\title{
Fracture Experiences and Long-Term Care Initiation among Older Population: Analysis of Korean National Health Insurance Service-Senior Cohort Study
}

\author{
Hye-jin $\mathrm{Kim}^{1}$, Soong-nang Jang ${ }^{1}$, Ja-kyung Lee ${ }^{1}$, Yong-Chan $\mathrm{Ha}^{2}$ \\ ${ }^{1}$ Red Cross College of Nursing, Chung-Ang University, Seoul, Korea \\ ${ }^{2}$ Department of Orthopaedic Surgery, Chung-Ang University College of Medicine, Seoul, Korea
}

Corresponding Author:

Soong-nang Jang, $\mathrm{MPH}, \mathrm{PhD}$

Red Cross College of Nursing, Chung-

Ang University, 84 Heukseok-ro,

Dongjak-gu, Seoul 06974, Korea

E-mail: sjang@cau.ac.kr

ORCID:

https://orcid.org/0000-0003-2621-945X

Received: May 12, 2019

Revised: July 27, 2019

Accepted: August 9, 2019
Background: Long-term care is a burden on individuals, families, and society. It is important to find ways to delay the onset of disability to lessen the burden of long-term care in aging societies. Fracture is one of the risk factors that affect physical functions and make older people dependent. This study aimed to examine how much more often older adults who experienced fractures initiated long-term care compared to those who did not, and whether the risk of entering long-term care differed significantly by fracture site. Methods: The analyses included insurants aged 65 years and over from the Korean National Health Insurance Service-senior cohort study (2002-2013). Cox proportional hazard models were used to calculate the hazard ratios of the first certification of initiation of long-term care after fracture, by fracture site, and for multiple recurrent fractures. Results: The incidence rate of initial long-term care beneficiaries was approximately 2.5 times higher when older people had experienced fractures; these individuals entered long-term care beneficiary status 3 years earlier compared to those who had no fracture events. Lower extremity fracture and multiple recurrent fractures more than doubled the risk for long-term care. Conclusion: Additional attention to fracture sites in prevention and rehabilitation settings is warranted to reduce disability and the related long-term care burden.

Key Words: Fractures, Long-term care, Survival analysis, Frail elderly

\section{INTRODUCTION}

Healthy aging policies must be established to maintain the health status of older adults and delay the initiation of long-term care (LTC) for as long as possible. Functional decline is one of the main factors for entering LTC; however, a preceding factor for healthy aging is the resilience of disease or injuries inducing disability. ${ }^{1}$ ) Fractures significantly affect normal functioning in older adults. It is especially difficult to recover from fractures after surgery and treatment. A vicious cycle begins, as hip fractures increase a patient's dependency, leading to even more falls. ${ }^{2,3)}$ In addition, limb injuries caused by fractures reduce social activity, productivity, and subsequently, cognitive stimulation to increase the risk of demen- tia and compromise life expectancy and quality of life. ${ }^{4-6)}$ Injuries, including fractures and dementia, independently increase the demand for LTC and also tend to be associated with one another or occur at the same time. ${ }^{7)}$ This synergistic association may lead to a sudden need for LTC.

A study in Germany predicted that disability and increased dependency caused by extremity injuries would increase the incidence of initiation of LTC among older adults. ${ }^{8)}$ The incidence rate of LTC is 2.5 times higher in older adults with extremity injuries and even higher for both lower and upper extremity and severe injuries. ${ }^{8)}$ However, we do not know the net long-term effect of fracture on care needs among the older population. It is particularly important to elaborate on the impact of geriatric fall-related inju- 
ries because fractures account for a high proportion of deaths from injuries. ${ }^{9)}$ Assessing the long-term health effects and care needs of geriatric fractures will help focus interventions on fractures and fall prevention in older populations. However, few studies have assessed the impact of fracture on LTC initiation. Since fracture experience is a life event that can have a sustained influence on body integration, balance, strength, and pain in older individuals, the present study investigated whether the long-term health (or functional) effect of fractures was linked to future dependency.

Accurate evaluation of the impact of fracture events in older adults on the incidence rate of the first certified use of LTC services, the social impact of LTC and fracture prevention policies can be concretely visualized. This study aimed to address the following questions using Korean national cohort data based on National Insurance claim datasets of older adults: is there an impact on the incidence rate of LTC initiation according to older adults' fracture experience and fracture sites? If yes, what is the magnitude of this impact?

\section{MATERIALS AND METHODS}

\section{Data and Sample}

We used the Korean National Health Insurance Service-senior sample cohort (NHIS-Senior) data to identify patients with fractures and determine the risk of LTC initiation in older adults. The Korean NHIS-Senior data covered a $10 \%$ random sample $(\mathrm{n}=558,147)$ of $5,500,000$ total insurants aged 60 years or above from 2002 to 2013. As national health insurance in Korea is mandatory, the NHIS-Senior sample is nationally representative. The cohort comprises five databases on participants' health insurance eligibility (general characteristics; age, sex, etc.), medical treatments, medical care institutions, health examinations, and LTC utilization. As the national LTC insurance system for older adults began in 2008, the database for LTC utilization has only been established since 2008 (2008-2013), while the other four databases (health insurance eligibility, medical treatments, medical care institutions, and health examinations) were established in 2002. Since its establishment in July 2008, LTC insurance system data were collected every December during the study period. Thus, data on LTC utilization in 2008 could be unclear.

After excluding individuals who were already in LTC in 2008, 447,276 older adults were identified as study samples in 2008 who did not enroll as LTC beneficiaries. They were followed for up to 12 years (2002-2013). The follow-up period (at least 1 year to a maximum of 11 years) varied depending on the occurrence of fractures (independent variables) and onset of LTC (dependent variables). The year of study entry was 2002 , and observation of frac- ture incidence (independent variable) was also launched in 2002. The study was reviewed and approved by the Institutional Review Board of Chung-Ang University (No. 1041078-201607-HR-14501K).

\section{Dependent variable}

We operationally defined 'LTC initiation' as receiving LTC insurance benefits for the first time. Beneficiaries of the LTC insurance schemes were individuals who were entered and registered into the LTC system. When assigned to one of three levels of LTC need through screening by the Care Needs Certification Board (including needs assessment), LTC benefits can be provided to applicants aged 65 years and above or geriatric patients under 65 years of age. According to the needs assessment and committee review, LTC services are provided ranging from level 3 (the lowest degree of care) to level 1 (the highest degree of care). The care need levels were further subdivided into five levels following the 2014 revision of the LTC insurance system. We aggregated levels 1-3 as LTC initiation, considering the consistency of the level of care needs. The first certification of LTC from 2009 to 2013 was our final outcome, which was considered the LTC initiation case ( 1 = LTC initiation, $0=$ no).

\section{Independent variables}

We defined fractures using claim data from the medical treatments database containing older adults' inpatient and outpatient diagnoses based on the 10th revision of the International Classification of Diseases (ICD-10) codes. Geriatric fall-related fractures fell into three categories based on the affected region: upper extremity fracture (ICD-10 codes: S52.5, S52.6, S42.2, S42.3), spine fracture (S22.0, S22.1, S32.0, M48.4, M48.5), and lower extremity fracture (S72.0, S72.1) (Table 1).

The fracture was a dummy variable, taking the value 1 if the fracture first occurred from 2002 onwards regardless of the fracture frequency, until 1 year before LTC initiation and 0 otherwise (cases without LTC initiation were followed until death or censoring). Regarding LTC initiation time, as we had the information only on the year of LTC initiation, we could not clarify whether fracture occurred before LTC initiation when they occurred within the same year (for example, when LTC initiation time was 2013, the fracture experience had to be observed from 2002 to 2012). We categorized combined fracture sites if the older adults experienced multiple recurrent fractures at multiple events during the study period. 'All fracture' was defined as recurrent fractures in the upper or lower extremities and spine at the same time or sequentially.

We examined demographic and chronic disease variables to explain the characteristics of LTC initiation. The demographics in- 
Table 1. Categorization of fracture sites and matching ICD-10 codes

\begin{tabular}{lc}
\hline Categorized fracture sites & ICD-10 code \\
\hline Upper extremity fracture & \\
$\quad$ Distal radius & $\mathrm{S} 52.5$ \\
$\quad$ Fracture of the distal radius & $\mathrm{S} 52.6$ \\
$\quad$ Combined fracture of the distal radius & \\
Humerus & $\mathrm{S} 42.2$ \\
$\quad$ Fracture of the proximal humerus & $\mathrm{S} 42.3$ \\
$\quad$ Fracture of shaft of humerus & \\
Spine fracture & $\mathrm{S} 22.0$ \\
Fracture of the thoracic spine & $\mathrm{S} 22.1$ \\
Multiple fractures of the thoracic spine & $\mathrm{S} 32.0$ \\
Fracture of the lumbar spine & $\mathrm{M} 48.4$ \\
Fatigue fracture of vertebra & $\mathrm{M} 48.5$ \\
Collapsed vertebra & \\
Lower extremity fracture & $\mathrm{S} 72.0$ \\
Fracture of the femoral neck & $\mathrm{S} 72.1$ \\
Pertrochanteric fracture &
\end{tabular}

ICD-10, International Classification of Diseases, 10th Revision.

cluded age and sex. As the age of individuals in the cohort began at 67 years, age was grouped into four categories (67-74, 75-84, 8594 , and $95+$ years). Common chronic diseases were included as dummy variables, taking the value 1 if the diseases first occurred from 2002 onwards until 1 year before LTC initiation and 0 otherwise. We determined the chronic diseases used as covariates after consulting with four medical doctors specialized in orthopedic surgery, rehabilitation medicine, and geriatric medicine. The chronic diseases included hypertension (ICD-10 codes: I10-I15), diabetes (E10-E14), ischemic diseases (I20-I25), cerebral diseases (I60-I69), hypercholesterolemia (E780), atrial fibrillation (I48), heart insufficiency (I50), lung insufficiency (J44), nervous diseases (including Parkinson disease; G20-G22), gastric diseases (K0$\mathrm{K} 9$ ), alcoholic liver disease (K70), atherosclerosis (I70), pneumonia (J12-J18), infections or parasites (A-B), external injuries (S-T, $\mathrm{V}-\mathrm{Y}$ ), dementia-Alzheimer disease, vascular dementia, Lewy body dementia, circumscribed brain atrophy, dementia as a side-effect of another disease, others not specified as dementia (F00/G30, F01, G31.82, G31.0, F02, F05.1, G23.1, F03), cancer (C00-C97), osteoporosis (M80, M81, M82), arthritis (osteoarthritis, rheumatoid arthritis; M15-M19, M05, M06), orthostatic hypotension (I95.1), chronic kidney disease (N18), urinary incontinence (N39.3, N39.4, N39.40, N39.41, N39.48), and depressive disorder (F32, F33).

\section{Statistical Analysis}

We performed descriptive statistics using frequency and percentage of the total study population. Regarding the characteristics of
LTC initiation cases, person-year exposures and the incidence rate of LTC initiation per 100 person-years were calculated. Kaplan-Meier survival curves and Cox proportional hazard models were used to examine fractures and the risk of LTC initiation after adjusting for age, sex, and chronic diseases. A stratified log-rank test was used to compare LTC initiation among groups with different types of fractures by the affected sites. Fracture sites were categorized as upper extremity, lower extremity, and spine. The hazard ratios for each fracture site were calculated since fractures were recorded as multiple events. Multiple fracture sites were categorized as seven types: upper extremity fracture only, lower extremity fracture only, spine fracture only, upper extremity and spine fracture, upper and lower extremity fracture, spine and lower extremity fracture, and all three fracture types. LTC initiation was available annually but there was no information on 'day' or 'month'; therefore, the results were presented by yearly age. If death and LTC initiation occurred in the same year during the study period, LTC initiation was counted. Since we could not clarify whether fractures or other diseases occurred before LTC initiation when they occurred within the same year, records of fractures and other diseases were observed until 1 year prior to LTC initiation. The statistical definition for the censoring event was 'death or no LTC initiation' by the end of the study period, while the complete event was the 'year of first LTC initiation. The effects of age can affect not only dependent variables but also independent variables. The variance inflation factor (VIF) and condition index showed no age and fracture multicollinearity. Thus, we included all covariates in the Cox proportional hazard models. Analyses were performed using SAS version 9.4 (SAS Institute Inc., Cary, NC, USA).

\section{RESULTS}

Table 2 shows the exposure and incidence rates of LTC initiation by the demographic and disease characteristics of the participants starting from 2009 . Of the 447,276 individuals, $43 \%$ were men and $57 \%$ were women. Most (56.6\%) of their ages ranged from 75 to 84 years. The fracture experience rate was $9.8 \%$. Among chronic diseases, $21.9 \%$ of the participants had cerebral disease, $6.8 \%$ had dementia, 2.3\% had nervous diseases including Parkinson disease, and $11.1 \%$ had depressive disorders. A total of 71,706 older adults entered LTC between 2009 and 2013; among them, around 64\% were women and about $63 \%$ were aged $75-84$ years.

The incidence rate of LTC initiation was 4.6 cases per 100 persons (95\% confidence interval [CI], 4.6-4.6). The incidence of LTC initiation was higher among women (5.0) than men (4.0) and increased with age. The incidence was 13 times higher among the oldest age group (over 95 years; 26.0) compared to that in old- 
118 Hye-jin Kim, et al.

Table 2. Incidence rates of LTC initiation in the Korean National Health Insurance Service-senior cohort $(\mathrm{n}=447,276)$

\begin{tabular}{|c|c|c|c|c|}
\hline Variable & $\%$ & Exposure (person-year) & Case of LTC initiation & $\begin{array}{l}\text { Incidence rate per } 100 \\
\text { person-year }(95 \% \mathrm{CI})\end{array}$ \\
\hline Total & & $1,561,880$ & 71,706 & $4.6(4.6-4.6)$ \\
\hline \multicolumn{5}{|l|}{ Sex } \\
\hline Male & 43.0 & 650,553 & 25,839 & $4.0(3.9-4.0)$ \\
\hline Female & 57.0 & 911,327 & 45,867 & $5.0(5.0-5.1)$ \\
\hline \multicolumn{5}{|l|}{ Age (y) } \\
\hline $75-84$ & 56.6 & 899,253 & 45,331 & $5.0(5.0-5.1)$ \\
\hline $85-94$ & 4.9 & 51,990 & 12,204 & $23.5(23.1-23.9)$ \\
\hline $95+$ & 0.1 & 742 & 193 & $26.0(26.0-29.9)$ \\
\hline \multicolumn{5}{|l|}{ Fracture } \\
\hline No & 90.2 & $1,418,137$ & 57,012 & $4.0(4.0-4.1)$ \\
\hline Lower fracture & 1.7 & 15,758 & 2,863 & $18.2(17.5-18.8)$ \\
\hline \multicolumn{5}{|l|}{ Multiple fracture sites ${ }^{\dagger}$} \\
\hline Upper extremity only & 3.3 & 51,364 & 4,668 & $9.1(8.8-9.4)$ \\
\hline Spine only & 4.5 & 66,007 & 19,923 & $30.2(29.8-30.6)$ \\
\hline Lower extremity only & 1.3 & 16,233 & 5,647 & $34.8(33.9-35.7)$ \\
\hline Upper \& spine fracture & 0.4 & 5,462 & 1,709 & $31.3(29.8-32.8)$ \\
\hline Upper \& lower fracture & 0.1 & 1,658 & 641 & $38.7(35.8-41.7)$ \\
\hline Spine \& lower fracture & 0.2 & 2,582 & 946 & $36.6(34.4-39.0)$ \\
\hline All fractures & 0.0 & 437 & 171 & $39.1(33.6-45.3)$ \\
\hline No fracture & 90.2 & $1,418,137$ & 57,012 & $4.0(4.0-4.1)$ \\
\hline \multicolumn{5}{|l|}{ Cerebral disease } \\
\hline Yes & 2.3 & 31,903 & 6,276 & $19.7(19.2-20.2)$ \\
\hline \multicolumn{5}{|l|}{ Dementia } \\
\hline No & 93.2 & $1,470,367$ & 51,967 & $3.5(3.5-3.6)$ \\
\hline Yes & 6.8 & 91,513 & 19,739 & $21.6(21.3-21.9)$ \\
\hline \multicolumn{5}{|l|}{ Orthostatic hypotension } \\
\hline No & 99.8 & $1,558,994$ & 71,420 & $4.6(4.6-4.6)$ \\
\hline Yes & 0.2 & 2,886 & 286 & $9.9(8.8-11.1)$ \\
\hline \multicolumn{5}{|l|}{ Depressive disorder } \\
\hline No & 88.9 & $1,391,034$ & 56,985 & $4.1(4.1-4.1)$ \\
\hline Yes & 11.1 & 170,846 & 14,721 & $8.6(8.5-8.8)$ \\
\hline \multicolumn{5}{|l|}{ Hypertension } \\
\hline No & 37.5 & 589,487 & 11,365 & $1.9(1.9-2.0)$ \\
\hline Yes & 62.5 & 922,705 & 39,987 & $4.3(4.3-4.4)$ \\
\hline \multicolumn{5}{|l|}{ Diabetes mellitus } \\
\hline No & 66.3 & $1,043,049$ & 39,623 & $3.8(3.8-3.8)$ \\
\hline Yes & 33.7 & 518,831 & 32,083 & $6.2(6.1-6.3)$ \\
\hline \multicolumn{5}{|l|}{ Ischemic disease } \\
\hline No & 78.4 & $1,229,998$ & 51,743 & $4.2(4.2-4.2)$ \\
\hline
\end{tabular}

(Continued to the next page) 
Table 2. Continued

\begin{tabular}{|c|c|c|c|c|}
\hline Variable & $\%$ & Exposure (person-year) & Case of LTC initiation & $\begin{array}{l}\text { Incidence rate per } 100 \\
\text { person-year }(95 \% \mathrm{CI})\end{array}$ \\
\hline Yes & 21.6 & 331,882 & 19,963 & $6.0(5.9-6.1)$ \\
\hline \multicolumn{5}{|c|}{ Hypercholesterolemia } \\
\hline No & 90.5 & $1,408,553$ & 63,986 & $4.5(4.5-4.6)$ \\
\hline Yes & 9.5 & 153,327 & 7,720 & $5.0(4.9-5.2)$ \\
\hline \multicolumn{5}{|c|}{ Atrial fibrillation } \\
\hline No & 96.9 & $1,517,679$ & 67,785 & $4.5(4.4-4.5)$ \\
\hline Yes & 3.1 & 44,201 & 3,921 & $8.9(8.6-9.2)$ \\
\hline \multicolumn{5}{|c|}{ Heart insufficiency } \\
\hline No & 92.9 & $1,460,080$ & 62,042 & $4.3(4.2-4.3)$ \\
\hline Yes & 7.1 & 101,800 & 9,664 & $9.5(9.3-9.7)$ \\
\hline \multicolumn{5}{|c|}{ Lung insufficiency } \\
\hline No & 86.9 & $1,371,173$ & 58,369 & $4.3(4.2-4.3)$ \\
\hline Yes & 13.1 & 190,707 & 13,337 & $7.0(6.9-7.1)$ \\
\hline \multicolumn{5}{|c|}{ Gastric diseases } \\
\hline No & 9.6 & 144,842 & 6,279 & $4.3(4.2-4.4)$ \\
\hline Yes & 90.4 & $1,417,038$ & 65,427 & $4.6(4.6-4.7)$ \\
\hline \multicolumn{5}{|c|}{ Alcoholic liver disease } \\
\hline No & 95.8 & $1,499,032$ & 68,983 & $4.6(4.6-4.6)$ \\
\hline Yes & 4.2 & 62,848 & 2,723 & $4.3(4.2-4.5)$ \\
\hline \multicolumn{5}{|c|}{ Atherosclerosis } \\
\hline No & 95.8 & $1,496,230$ & 66,708 & $4.5(4.4-4.5)$ \\
\hline Yes & 4.2 & 65,650 & 4,998 & $7.6(7.4-7.8)$ \\
\hline \multicolumn{5}{|c|}{ Pneumonia } \\
\hline No & 85.3 & $1,343,355$ & 55,670 & $4.1(4.1-4.2)$ \\
\hline Yes & 14.7 & 218,525 & 16,036 & $7.3(7.2-7.5)$ \\
\hline \multicolumn{5}{|c|}{ Infections or parasites } \\
\hline No & 40.1 & 617,191 & 27,205 & $4.4(4.4-4.5)$ \\
\hline Yes & 59.9 & 944,689 & 44,501 & $4.7(4.7-4.8)$ \\
\hline \multicolumn{5}{|c|}{ External injury } \\
\hline No & 30.2 & 467,515 & 16,460 & $3.5(3.5-3.6)$ \\
\hline Yes & 69.8 & $1,094,365$ & 55,246 & $5.1(5.0-5.1)$ \\
\hline \multicolumn{5}{|l|}{ Cancer } \\
\hline No & 88.4 & $1,393,489$ & 60,050 & $4.3(4.3-4.3)$ \\
\hline Yes & 11.6 & 168,391 & 11,656 & $6.9(6.8-7.1)$ \\
\hline \multicolumn{5}{|c|}{ Osteoporosis } \\
\hline No & 70.7 & $1,095,837$ & 43,396 & $4.0(3.9-4.0)$ \\
\hline Yes & 29.3 & 466,043 & 28,310 & $6.1(6.0-6.2)$ \\
\hline \multicolumn{5}{|c|}{ Arthritis (osteoarthritis, rheumatoid arthritis) } \\
\hline No & 36.2 & 556,671 & 21,392 & $3.8(3.8-3.9)$ \\
\hline Yes & 63.8 & $1,005,209$ & 50,314 & $5.0(5.0-5.1)$ \\
\hline \multicolumn{5}{|c|}{ Urinary incontinence } \\
\hline No & 97.7 & $1,526,426$ & 68,370 & $4.5(4.5-4.5)$ \\
\hline Yes & 2.3 & 35,454 & 3,336 & $9.4(9.1-9.7)$ \\
\hline
\end{tabular}

LTC, long-term care; CI, confidence interval.

*Fracture experience at each site, multiple cases.

${ }^{+}$Combinations of fracture sites during the observation period.

er adults (67-74 years; 2.3$)$. Of the total cohort participants who entered LTC, around one in four cases experienced fracture $(n=14,694)$. The incidence of LTC initiation was 10.2 per 100 person-years, approximately 2.5 times higher among participants who experienced fractures compared to those who did not. Those who experienced upper extremity fractures entered LTC at 10.2 
A

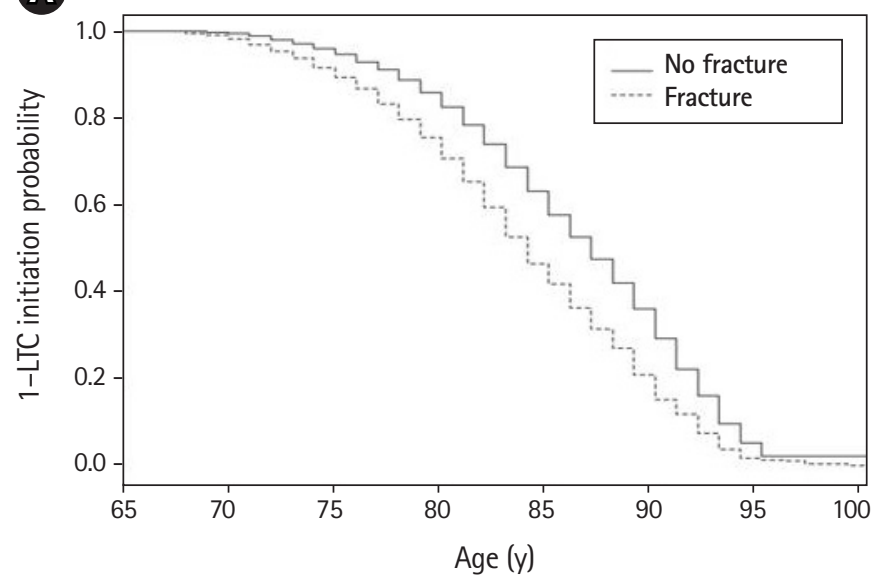

C

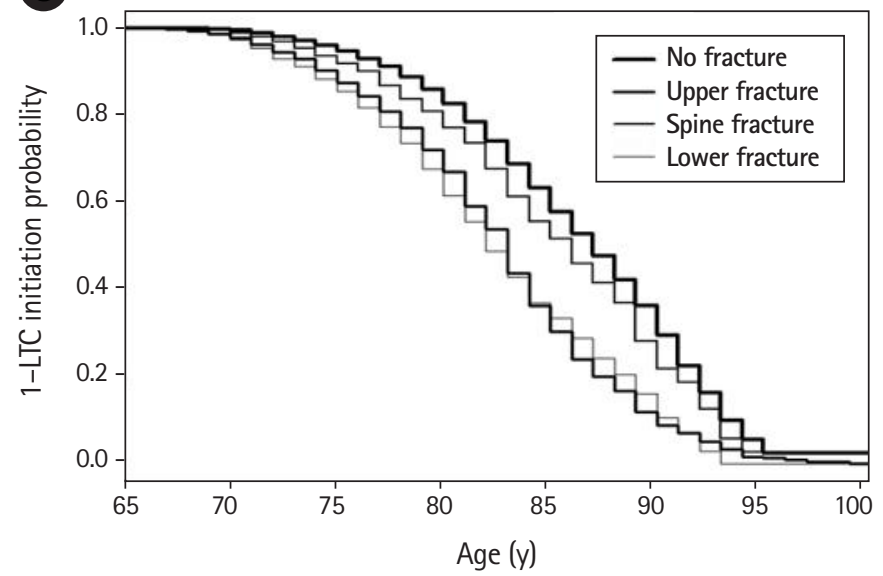

B

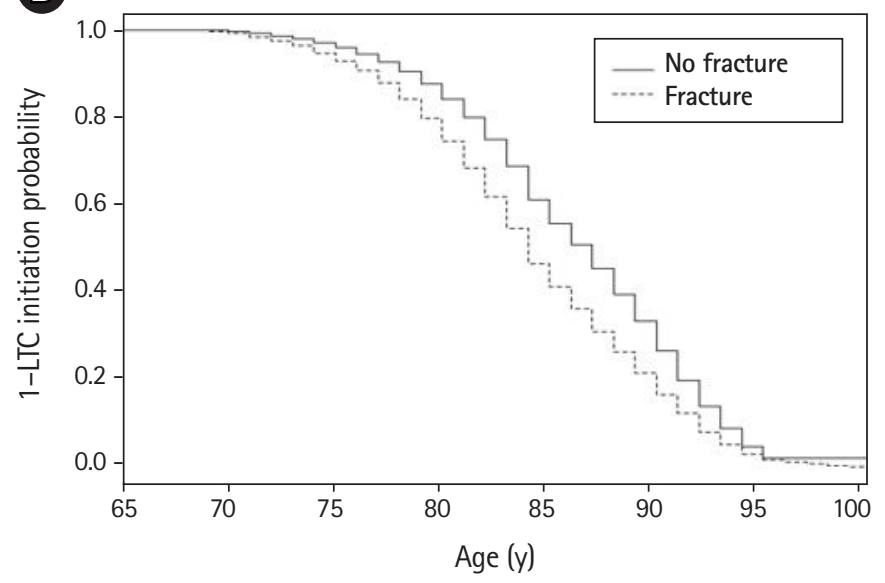

(D)

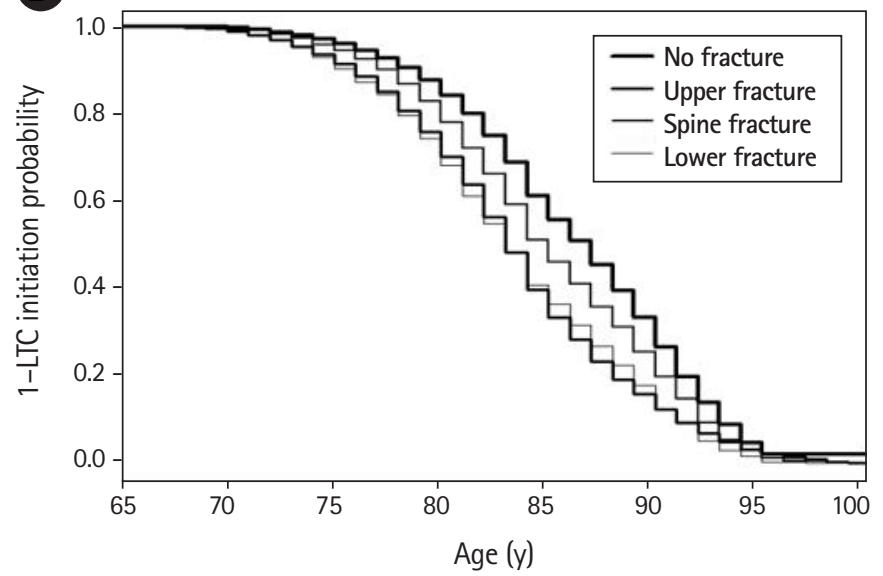

Fig. 1. Long-term care (LTC) initiation among age, sex, and fracture sites in the Korean National Health Insurance Service-senior sample cohort (Kaplan-Meier survival curves). LTC initiation by fracture in men (A) and women (B). LTC initiation by fracture sites in men (C) and women (D). Upper fracture means upper extremity fracture and lower fracture means lower extremity fracture.

cases per 100 person-years, while LTC initiation of lower extremity and spinal fractures were 18.2 and 8.8 cases per 100 person-years, respectively. The incidence rate of LTC initiation was $21.6 \%$ among older adults with dementia, $19.7 \%$ in those with nervous disease, and $11.1 \%$ in those with cerebral disease.

Fig. 1 shows the Kaplan-Meier curve of the incidence rate of LTC initiation depending on fracture experience and fracture sites according to sex. The incidence rate of LTC initiation was significantly higher among men and women who had previously experienced fractures than those without fractures. Half of all men and women required LTC around 87 years of age. In addition, half of the older adults with previous fractures entered LTC at age 84 compared to age 87 in people with no fracture history. Thus, older adults with fracture entered LTC around 3 years earlier than those without (Fig. 1A, 1B and Appendix 1). Men with upper or lower extremity fractures entered LTC around 3 years earlier than those with spine fractures, compared to 1 year earlier in women (Fig. 1C,
1D and Appendix 1). Moreover, men with fractures of all three sites (upper extremity, spine, and lower extremity) entered LTC 10 years earlier than those without any fractures, compared to 6 years earlier in women (Appendix 1).

Regarding the hazard ratio of LTC initiation by fracture experience, the hazard ratios increased 1.66-fold (95\% CI, 1.61-1.71), 1.36-fold (95\% CI, 1.32-1.40), and 2.25-fold (95\% CI, 2.17-2.33) for the upper extremity, spinal, and lower extremity fracture groups, respectively, compared to those in the non-fracture group after adjusting for age, sex, and chronic diseases. Among participants in the all-fracture group with multiple recurrent fractures of the upper extremity, spine, and lower extremity, the hazard ratio increased 3.21-fold (95\% CI, 2.71-3.81). Among those with chronic diseases, the hazard ratio of LTC initiation was highest for those with dementia (2.60; 95\% CI, 2.55-2.65) compared with those without dementia (Table 3 ). 
Table 3. Hazard ratios of long-term care initiation by age, sex, fracture site, and chronic disease

\begin{tabular}{lcc}
\hline Variable & aHR $(95 \% \mathrm{CI})$ & $\mathrm{p}$-value \\
\hline Age $(\mathrm{y})$ & Ref & \\
$67-74$ & $1.68(1.65-1.71)$ & $<0.0001$ \\
$75-84$ & $6.33(6.17-6.49)$ & $<0.0001$ \\
$85-94$ & $6.28(5.44-7.24)$ & $<0.0001$ \\
$95+$ & & \\
Gender & Ref & \\
Male & $1.03(1.01-1.05)$ & 0.0035 \\
Female & & \\
Multiple fracture sites & 1 & \\
No fracture & $1.66(1.61-1.71)$ & $<0.0001$ \\
Upper extremity & $1.36(1.32-1.40)$ & $<0.0001$ \\
Spine extremity & $2.25(2.17-2.33)$ & $<0.0001$ \\
Lower extremity & $2.03(1.89-2.18)$ & $<0.0001$ \\
Upper extremity \& spine & $2.97(2.70-3.26)$ & $<0.0001$ \\
Upper \& lower extremity & $2.12(1.94-2.31)$ & $<0.0001$ \\
Spine \& lower extremity & $3.21(2.71-3.81)$ & $<0.0001$ \\
All fractures & & \\
\hline
\end{tabular}

aHR, adjusted hazard ratio; CI, confidence interval.

The Cox proportional hazard model was adjusted for 22 chronic diseases as covariates.

\section{DISCUSSION}

The results of our analyses revealed three sets of patterns associated with fracture experience and LTC initiation in the Korean older population. First, fracture was associated with the probability of becoming an LTC recipient, increasing from 1.66- to 3.21-fold according to the fracture site within 10 years. In other words, those who experienced fractures entered LTC 3 years earlier. Secondly, the results varied according to the fracture site. The probability of becoming an LTC recipient was more than two times higher in those who had at least one lower extremity fracture. Thirdly, the variety of fracture sites was also important. Experiencing multiple recurrent fractures in both upper, lower extremity and spine increased the risk of entering LTC by more than 3-fold compared to the risk in the non-fracture group and was higher than the risk of entering LTC in patients with dementia. These results confirmed that the long-term outcome in patients with fractures differs by fracture site and multiple fracture sites. Given that the national LTC expenditure is about $\$ 4$ billion in Korea, ${ }^{10)}$ older individuals entering LTC 3 years earlier may significantly increase the LTC cost burden.

LTC initiation was significantly higher for lower extremity fractures. Hip fractures undermine disability-adjusted life years in older adults, thereby increasing disease burdens. This may be because lower extremity fractures lead to mobility limitation. ${ }^{8,11)}$ Indeed, the likelihood of returning to the previous level of mobility is low in hip fracture patients. Only 34\% of older adults who sustained a hip fracture returned to pre-fracture mobility function. ${ }^{12)}$ Physical activity, balance, and mobility have been repeatedly demonstrated to be beneficial for improving physical function in older adults ${ }^{13)}$ in terms of short-term and long-term effects. ${ }^{14,15)}$ These results support the efficacy of preventing lower extremity fracture and restoring function and mobility to pre-fracture levels as to delay the need for LTC.

Multiple recurrent fractures in various body parts proved to be a stronger marker than dementia of a future need for LTC. This fracture-prone condition can be classified into high fall-risk, frail, and/ or osteoporotic status in old ages. In this condition, a vicious cycle begins in which the slow recovery from fractures due to a fall and increased dependency lead to additional falls. ${ }^{2,3,16)}$ To prevent multiple recurrent fractures among older adults, bone health promotion, fall prevention, and management after fall incidence must be performed simultaneously. In addition, comorbidity including osteoporosis, dementia, and other diseases must be considered. ${ }^{17)}$ Fractures can also give rise to neurodegenerative diseases such as Parkinson disease and dementia (a major factor of LTC initiation), which may indirectly enhance care needs. A decrease in social activity due to fracture can also reduce cognitive stimulation and act as a risk factor for dementia. ${ }^{5)}$ The study in Germany reported a higher rate of LTC initiation in people who experienced both dementia and extremity injuries compared to that in those who experienced each disease independently. ${ }^{8)}$ Dementia may affect fractures and is a major factor associated with an increased rate of hospitalization due to fracture. ${ }^{18-20)}$

The difference in LTC initiation by fracture site was shared between men and women. However, the Kaplan-Meir curves showed a sex difference in the age of LTC onset. Men with extremity fractures entered LTC about 3 years earlier than those with spine fracture, while women entered LTC only 1 year earlier. In addition, participants with diverse fracture sites also became LTC recipients much earlier. Men with fractures of the upper and lower extremities and spine, regardless of whether they occurred simultaneously, became LTC recipients 10 years earlier than men without any fractures, compared to 6 years earlier in women. This result suggests that the fracture severity might be higher in men than in women. Several studies also reported higher mortality and institutionalization rates after hip fracture in men than in women. ${ }^{21-23)}$ Our results add to this evidence by showing sex differences and the exact degrees of risk for initiating LTC in men and women.

The enormous individual and social burdens due to LTC in older adults may be reduced through interventions addressing the risk factors for lower extremity and multiple recurrent fractures. Inter- 
ventions targeting fracture prevention and rehabilitation such as exercise therapy and environmental management are effective in terms of disability and physical function. ${ }^{2426)}$ These interventions should also consider sex differences in the impact of fractures.

Several limitations warrant consideration in generalizing our observations. First, since the National Health Insurance Service provides only limited datasets retrieved from insurance claim databases, analyses were performed for LTC initiation in a limited time. The data lacked information on socio-economic status, health behaviors, fall incidence, and other risk factors for fractures and LTC needs. Second, LTC initiation was only considered if it occurred within the years after the fracture. If the fracture was the reason for obtaining benefits from LTC insurance and occurred within the first 6 months, the information on the time of LTC initiation might be not accurate. Fractures that occurred 2-3 years before the first onset of LTC may be more closely related to the cause of disability, especially for cases requiring hospitalization. Consideration should be given to when the fracture occurred, and it is also necessary to distinguish between hospitalization and outpatient use after fractures. Third, although the use of medications can increase the risk of falls and fractures through various mechanisms and also affect LTC initiation, we excluded drug data. The overreporting of geriatric-related fractures might be due to the inclusion of the accident-driven fractures. Moreover, there was a lack of reliable information on fragility fractures based on clinical data.

In conclusion, fractures led to a nearly 2.5 -fold increase in the risk of LTC initiation among older adults even after adjusting for chronic diseases in the model. To our knowledge, this is the first longitudinal study to report the association between fracture sites and LTC initiation using a nationally representative cohort data from older adults in Korea. A strong emphasis is needed on the prevention of geriatric-related fractures as a top priority strategy in actions to delay the initiation of LTC; it is important to build on the resilience of the older population and a sustainable society. In order to delay the onset of disability and to maintain independent lives as long as possible, prevention of fracture and sufficient rehabilitation care after fracture must be considered key interventions in LTC policies.

\section{CONFLICT OF INTEREST DISCLOSURES}

The researchers claim no conflicts of interest.

\section{ACKNOWLEDGEMENTS}

This work was supported by the Chung-Ang University Research Scholarship Grants in 2017, and the Korea Health Technology
R\&D Project through the Korea Health Industry Development Institute funded by the Ministry of Health \& Welfare, Republic of Korea (No. HC15C1189). This study used the administrative claims data from Korean National Health Insurance Service (No. NHIS-2017-2-362). Data access was legally approved by Korean National Health Insurance Service and individual patients cannot be identified. The sponsors and Korean National Health Insurance Service had no role in this study.

\section{REFERENCES}

1. Beard JR, Officer A, de Carvalho IA, Sadana R, Pot AM, Michel JP, et al. The world report on ageing and health: a policy framework for healthy ageing. Lancet 2016;387:2145-2154.

2. Handoll HH, Sherrington C, Mak JC. Interventions for improving mobility after hip fracture surgery in adults. Cochrane Database Syst Rev 2011;(3):CD001704.

3. Dyer SM, Crotty M, Fairhall N, Magaziner J, Beaupre LA, Cameron ID, et al. A critical review of the long-term disability outcomes following hip fracture. BMC Geriatr 2016;16:158.

4. Hamer M, Chida Y. Physical activity and risk of neurodegenerative disease: a systematic review of prospective evidence. Psychol Med 2009;39:3-11.

5. Lautenschlager NT, Cox K, Kurz AF. Physical activity and mild cognitive impairment and Alzheimer's disease. Curr Neurol Neurosci Rep 2010; 10:352-8.

6. Zhou Y, Putter H, Doblhammer G. Years of life lost due to lower extremity injury in association with dementia, and care need: a 6-year follow-up population-based study using a multi-state approach among German elderly. BMC Geriatr 2016;16:9.

7. de Bruijn RF, Schrijvers EM, de Groot KA, Witteman JC, Hofman A, Franco OH, et al. The association between physical activity and dementia in an elderly population: the Rotterdam Study. Eur J Epidemiol 2013;28:277-83.

8. Barth A, Vatterrott A, Zhou Y, Fink A, Doblhammer G. Extremity injuries and dementia disproportionately increase the risk for long-term care at older age in an analysis of German Health Insurance routine data for the years 2006 to 2010. Eur Rev Aging Phys Act 2016;13:9.

9.Haagsma JA, Graetz N, Bolliger I, Naghavi M, Higashi H, Mullany EC, et al. The global burden of injury: incidence, mortality, disability-adjusted life years and time trends from the Global Burden of Disease study 2013. Inj Prev 2016;22:3-18.

10. National Health Insurance Service. Long-term care insurance statistical yearbook. Wonju: National Health Insurance Service; 2016.

11. Papadimitriou N, Tsilidis KK, Orfanos P, Benetou V, Ntzani EE, 
Soerjomataram I, et al. Burden of hip fracture using disability-adjusted life-years: a pooled analysis of prospective cohorts in the CHANCES consortium. Lancet Public Health 2017;2: e239-e246.

12. Tang VL, Sudore R, Cenzer IS, Boscardin WJ, Smith A, Ritchie $\mathrm{C}$, et al. Rates of recovery to pre-fracture function in older persons with hip fracture: an observational study.J Gen Intern Med 2017;32:153-8.

13. Centers for Disease Control and Prevention. The state of aging and health in America 2013. Atlanta: Centers for Disease Control and Prevention; 2013.

14. Rejeski WJ, Marsh AP, Chmelo E, Prescott AJ, Dobrosielski M, Walkup MP, et al. The Lifestyle Interventions and Independence for Elders Pilot (LIFE-P): 2-year follow-up.J Gerontol A Biol Sci Med Sci 2009;64:462-7.

15. Roberts S, Awick E, Fanning JT, Ehlers D, Motl RW, McAuley E. Long-term maintenance of physical function in older adults following a DVD-delivered exercise intervention. J Aging Phys Act 2017;25:27-31.

16. Harvey L, Mitchell R, Brodaty H, Draper B, Close J. The influence of dementia on injury-related hospitalisations and outcomes in older adults. Injury 2016;47:226-34.

17. Harvey NC, McCloskey EV, Mitchell PJ, Dawson-Hughes B, Pierroz DD, Reginster JY, et al. Mind the (treatment) gap: a global perspective on current and future strategies for prevention of fragility fractures. Osteoporos Int 2017;28:1507-29.

18. Harvey L, Mitchell R, Brodaty H, Draper B, Close J. Differing trends in fall-related fracture and non-fracture injuries in older people with and without dementia. Arch Gerontol Geriatr 2016;67:61-7.

19. Li F. Alzheimer's disease increases the incidence of hospitaliza- tion due to fall-related bone fracture in elderly Chinese. Int J Gerontol 2016;10:227-31.

20. Fernando E, Fraser M, Hendriksen J, Kim CH, Muir-Hunter SW. Risk factors associated with falls in older adults with dementia: a systematic review. Physiother Can 2017;69:161-70.

21. Fransen M, Woodward M, Norton R, Robinson E, Butler M, Campbell AJ. Excess mortality or institutionalization after hip fracture: men are at greater risk than women. J Am Geriatr Soc 2002;50:685-90.

22. Trombetti A, Herrmann F, Hoffmeyer P, Schurch MA, Bonjour JP, Rizzoli R. Survival and potential years of life lost after hip fracture in men and age-matched women. Osteoporos Int 2002; 13:731-7.

23. Endo Y, Aharonoff GB, Zuckerman JD, Egol KA, Koval KJ. Gender differences in patients with hip fracture: a greater risk of morbidity and mortality in men.J Orthop Trauma 2005;19:29-35.

24. Sherrington C, Fairhall N, Kirkham C, Clemson L, Howard K, Vogler C, et al. Exercise and fall prevention self-management to reduce mobility-related disability and falls after fall-related lower limb fracture in older people: protocol for the RESTORE (Recovery Exercises and STepping On afteR fracturE) randomised controlled trial. BMC Geriatr 2016;16:34.

25. Turunen K, Salpakoski A, Edgren J, Tormakangas T, Arkela M, Kallinen M, et al. Physical activity after a hip fracture: effect of a multicomponent home-based rehabilitation program: a secondary analysis of a randomized controlled trial. Arch Phys Med Rehabil 2017;98:981-8.

26. Lim JY. Editorial: Fragility fracture care: an urgent need to implement the integrated model of geriatric care. Ann Geriatr Med Res 2019;23:1-2. 
124 Hye-jin Kim, et al.

Appendix 1. Age (y) of long-term care initiation from life tables of survival analysis

\begin{tabular}{|c|c|c|c|c|c|c|}
\hline & \multicolumn{2}{|c|}{$25 \%$ initiation } & \multicolumn{2}{|c|}{$50 \%$ initiation } & \multicolumn{2}{|c|}{$75 \%$ initiation } \\
\hline & Men & Women & Men & Women & Men & Women \\
\hline No fracture & 82 & 82 & 87 & 87 & 91 & 91 \\
\hline Upper extremity fracture only & 79 & 80 & 83 & 84 & 87 & 87 \\
\hline Spine fracture only & 82 & 81 & 86 & 85 & 90 & 90 \\
\hline Lower extremity fracture only & 78 & 80 & 83 & 84 & 88 & 88 \\
\hline Upper extremity \& spine fracture & 76 & 79 & 83 & 83 & 84 & 86 \\
\hline Upper extremity \& lower extremity fracture & 77 & 78 & 81 & 82 & 84 & 86 \\
\hline Spine \& lower extremity fracture & 79 & 79 & 82 & 83 & 89 & 88 \\
\hline All fractures & 73 & 78 & 77 & 81 & 84 & 85 \\
\hline
\end{tabular}

